\title{
Maximal singular integrals
}

\author{
Nicolas Th. VAROpoulos
}

\begin{abstract}
We prove the $L_{p}$ boundedness of the maximal operators attached to the singular kernels introduced in [1]. These kernels are obtained by multiplying (pointwise) a classical convolution Calderon-Zygmund kernel with the perturbing factor $[a]_{x, y}$ ( $c f$. below). The importance of these perturbations lies in potential theoretic applications ( $c f .[3,7])$.
\end{abstract}

Mathematics Subject Classification (2000): 42B20 (primary); 42B25 (secondary).

\section{Introduction}

\subsection{Notation and statement of the theorem}

In this note I shall examine maximal operators that are related to the singular integrals that were introduced in [1] ( $c f$. also [7]). I shall preserve the notation of [1,7] and denote $[a]_{x, y}=\int_{0}^{1} a(x+t(y-x)) d t ; x, y \in \mathbb{R}^{n}, a \in C_{0}^{\infty}\left(\mathbb{R}^{n}\right)$. I shall also use the abbreviation $F\left([a]_{x, y}\right)=F\left(\left[a_{1}\right]_{x, y}, \cdots,\left[a_{k}\right]_{x, y}\right)$ for $a_{1}, \cdots, a_{k} \in$ $C_{0}^{\infty}$ and where $F\left(z_{1}, \cdots, z_{k}\right) \in C^{N}(N \geq 1)$ will denote throughout some sufficiently smooth function of $k$ complex variables. We shall denote throughout by $\Omega \in L^{r}(\Sigma)(r>1)$ where $\Sigma=\left[x \in \mathbb{R}^{n} ;|x|=1\right]$ is the unit sphere and where $L^{r}$ or $L_{r}$ denotes throughout the Lebesgue space on the appropriate measure space. The condition $\int \Omega=0$ will be assumed throughout.

We shall be concerned in this paper with the following kernel and the corresponding principal value operator and the associated maximal function:

$$
\begin{gathered}
K(x, y)=\Omega\left(\frac{x-y}{|x-y|}\right)|x-y|^{-n} F\left([a]_{x, y}\right) ; x, y \in \mathbb{R}^{n}, \\
f \longmapsto K_{\epsilon, M} f(x)=\int_{\varepsilon<|x-y|<M} K(x, y) f(y) d y, \\
K^{*} f(x)=\sup _{\epsilon, M}\left|K_{\epsilon, M} f(x)\right| ; f, a_{j} \in C_{0}^{\infty}, 1 \leq j \leq k .
\end{gathered}
$$


In [1] the authors consider kernels that are more general, but also smoother:

$$
T(x, y)=L(x-y) F\left([a]_{x, y}\right),
$$

where $L$ is a classical convolution Calderon-Zygmund kernel (i.e. $\widehat{L} \in L^{\infty},|L(x)| \leq$ $C|x|^{-n}$, and $|L(x+h)-L(x)| \leq C|h|^{\varepsilon}|x|^{-n-\varepsilon}$ for $\left.2|h| \leq|x|\right)$. It is then proved in [1] that the corresponding principal value operator $T_{\varepsilon, M}$ is $L_{p} \rightarrow L_{p}$ bounded, uniformly in $\varepsilon, M$ for $1<p<\infty$, with bounds that only depend on $\|a\|_{\infty}$ and $\|F\|_{C^{N}}$ (for some $N \geq 1$ ). The formulation in [1] is slightly different, but equivalent: In [1] $F(z)=z^{p}(p=1,2, \cdots)$ but the operator norms are then $O\left(p^{N}\right)$ for some $N>0$.

The kernel $T$ is not a standard kernel ( $c f$. [1]) and therefore the general theory [6, Chapter I, 7.3] cannot be used to deduce the $L^{p}$ boundedness of the corresponding maximal operator (defined as in $(0.3)$ ).

In the notation I shall preserve the letters $c, C$, possibly with indices, to indicate positive constants that may differ from place to place but are independent from the main parameters of the formulaes.

Theorem 0.1. Let $\Omega, F, n, N, r$ be as above and let $a_{1}, \cdots, a_{k} \in C_{0}^{\infty}$ be such that $\left\|a_{j}\right\|_{\infty} \leq 1$. For every $1<p<\infty$ there exists $C$ depending on $n, N, r, k, p$, but not on the $a_{j}$ 's, such that

$$
\left\|K^{*} f\right\|_{p} \leq C\|\Omega\|_{r}\|F\|_{C^{N}}\|f\|_{p} ; f \in C_{0}^{\infty}\left(\mathbb{R}^{n}\right) .
$$

\|\|$_{p}$ indicates throughout the corresponding $L_{p}$ norm.

The estimate (0.4) with $K^{*}$ replaced by $K_{\varepsilon, M}$ (but uniform in $\epsilon, M$ ) is in [7, Corollary, Section 1.3] ( $c f$. [1] for the case $\Omega \in C^{m}$ ). As I already pointed out in [7] the integrability of $\Omega$ can be improved to $\Omega \epsilon L\left(\log ^{+} L\right)^{a}$ for some $a \geq 1$. It is easy to see that the same $a \geq 1$ that works in [7] also works in the above Theorem. When $\Omega$ is odd ( 0.4$)$ follows by the method of rotation.

The critical first part of the proof of the theorem for $p=2$ only uses the uniform bound of $\left\|K_{\epsilon, M}\right\|_{2 \rightarrow 2}$ and the additional key estimate [7, (1.32)]. For the more general $1<p<\infty$ some additional notions from [1,7] will be needed, but none of the technical aspects of these papers. Some of these facts from [7] will be elaborated in the Appendix at the end of the paper. As a result familiarity with $[1,7]$ although desirable, is not essential for the understanding of the first part of the proof.

The weak $L^{1}$ boundedness of all the above operators, even in the smooth case in the setting of [1], remains an open (and interesting) problem.

\subsection{Further notation and plan of the proof}

We say that a function $A(x, h)$ on $\mathbb{R}_{+}^{n+1}=\left(x \in \mathbb{R}^{n}, h>0\right)$ lies in $D_{p},(0<$ $p<\infty)$ if the non tangential maximal function $A^{*}$ and $S$-function: $S(h \nabla A)(x)=$ $\left(\iint_{\Gamma(x)}|h \nabla A|^{2} \frac{d x d h}{h^{n+1}}\right)^{\frac{1}{2}}$ both lie in $L^{p}\left(\mathbb{R}^{n}\right)$ (Here $\Gamma(x) \subset \mathbb{R}_{+}^{n+1}$ denotes the conical 
wedge with vertex at $x \in \mathbb{R}^{n}$ ). These spaces were introduced for the first time in [3]. $C f$. [2,7] for further elaborations and for the case $p=\infty$.

Let now $Q(x, y ; h) \in L_{\text {loc }}^{1}\left(x, y \in \mathbb{R}^{n}, h>0\right)$ and let us define the bilinear form: (cf. [7, Section 2.5])

$$
B(A, f)=\int Q(x, y ; h) f(x) A(y, h) \frac{d x d y d h}{h} ; A, f \in C_{0}^{\infty} .
$$

A mapping $L: C_{0}^{\infty}\left(\mathbb{R}_{+}^{n+1}\right) \longrightarrow \mathcal{D}^{\prime}\left(\mathbb{R}^{n}\right)$ (= the space of distribution on $\mathbb{R}^{n}$ ) can then be defined by

$$
(L A, \varphi)=B(A, \varphi) ; \varphi \in C_{0}^{\infty}\left(\mathbb{R}^{n}\right)
$$

The kernels $Q$ that we shall consider in this paper $(c f .[7,(1.19)]$ are of the form:

$$
Q(x, y ; h)=Q_{h}(x-y) F(x, y ; h)=\Omega\left(\frac{x-y}{|x-y|}\right) \theta_{h}(|x-y|) F(x, y ; h),
$$

where $F$ is a perturbing factor and where in the convolution kernel $\Omega \in L^{r}$ with $\int \Omega=0$ is as in the theorem, and $\theta \in C_{0}^{\infty}$ with supp $\theta \subset[1,2]$.

The standard notation $Q_{h}(x)=h^{-n} Q\left(\frac{x}{h}\right)(h>0)$ as well as the notation $Q_{h} f(x)=\int Q_{h}(x-y) f(y) d y\left(f \in C_{0}^{\infty}\right)$ will also be used. I shall also use a notation that proved very convenient in [7]; this consists in reserving the letter $\check{P}$ for the elements of $C_{0}^{\infty}\left(\mathbb{R}^{n}\right)$ and the letter $\check{Q}$ for the elements of $C_{00}^{\infty}$, i.e. when in addition $\int \check{Q}=0$. These $\check{P}, \check{Q}$ differ from place to place. Furthermore in (0.7) we shall set $F=F\left([a]_{x, y}\right)$ unless otherwise stated. We shall also denote $F_{0}=c_{0}+\sum_{i=1}^{k} c_{i} z_{i}$ the affine functions. Together with $L$ in $(0.6),(0.7)$ I shall define the maximal operator

$$
L^{*} A(x)=\sup _{\epsilon, M}\left|\int_{\epsilon<h<M} Q(x, y ; h) A(y, h) \frac{d y d h}{h}\right| ; A \in C_{0}^{\infty},
$$

and the essential step in the proof of the theorem is the following.

Proposition 0.2. With $Q, p, a_{j}$ and $F$ as in the theorem we have

$$
\left\|L^{*} A\right\|_{p} \leq C\|\Omega\|_{r}\|F\|_{C^{N}}\|A\|_{D_{p}}
$$

with $C=C(n, p, k, N, r)$.

\|\|$_{D_{p}}$ indicates the natural norm in the $D_{p}$-spaces. The proof of this proposition is given in Sections 1-3 below and it is the crucial part of the paper. 
Modulo classical maximal functions on the other hand (cf. [7, (1.20) (1.21)] the theorem is equivalent to the $L^{p} \longrightarrow L^{p}$ boundedness of the operator

$$
f \mapsto \sup _{\epsilon, M}\left|\int_{\epsilon<h<M} Q(x, y ; h) f(y) \frac{d y d h}{h}\right| ; f \in C_{0}^{\infty} .
$$

But for $f \in L^{p}$ we have $\check{P}_{h} f(y) \in D_{p},(1<p<\infty, c f$. [2,6]) and Proposition 0.2 applies. For (0.10) it suffices therefore to examine the correcting maximal operator where in the integral we replace $f(y)$ by $f(y)-\check{P}_{h} f(y)$. This is done in the second part of the paper in Section 4-5 and uses a Cotlar analysis and some other ideas from [1]. The estimate of this correcting term follows very closely [7, Section 8-9]. Adapting the arguments of [7], is straightforward indeed for $p=2$. For the general case $p \neq 2$ I shall go back and elaborate on the arguments of the last subsection of [7, Section 9] because not many details were given there. This second part of the proof will be difficult to read without a reasonable familiarity of [7, Section 8-9]. I should point out however that, although technical, this second part of the proof uses either classical tools, or ideas that have been borrowed from [1].

\subsection{The smooth case}

If we assume that $\Omega \in C^{\alpha}$ (some $\alpha>0$ ) in (0.1) - this a special case of [1, $\left.(0.1)^{\prime}\right]$ - a different, much shorter, proof of the Proposition 0.2 can be given. This proof avoids altogether the difficult estimate [7, (1.32)], but uses instead the vector valued (i.e. valued in $\ell^{2}$ ) version of the generalized $\mathrm{T} 1$ theorem that I developed in [8].

Using that version of the T1 theorem we can prove the critical case of Proposition 0.2 when $F$ is an affine function. More precisely this proof gives Proposition 1.1 for $p=\infty$ (with $L^{p}\left(\ell^{2}\right)$ interpreted as the $\ell^{2}$-valued BMO). The other values of $p$ then follow by general considerations ( [7, Section 2.5]).

This approach is simpler (because it avoids [7, (1.32)]) but it has the drawback that it needs the smoothness of $\Omega$. I shall not give the details but the motivated reader, I am sure, will be able to adapt [8] to give this alternative proof of (1.6), below, in this special case. The starting point of this proof can be found in the remark at the end of Section 2.1 below.

\section{The general strategy for Proposition 0.2}

\subsection{Notation and the vector valued operator}

We shall fix once and for all $R \in C_{0}^{\infty}\left(\mathbb{R}^{n}\right)$ in such a way that the Fourier transform satisfies $|\widehat{R}(\xi)-1|=O\left(|\xi|^{a}\right)$ for some large $a \geq 1$. Let us also define the operators 
$L_{k}, \widetilde{L}_{N}$ with $L=L_{k}+\widetilde{L}_{2^{k}}$ by truncating the definition (0.6):

$$
\begin{aligned}
\left(L_{k} A, f\right) & =\int_{h>2^{k}} Q(x, y ; h) f(x) A(y, h) \frac{d x d y d h}{h}, \\
\left(\widetilde{L}_{N} A, f\right) & =\int_{h<N} Q(x, y ; h) f(x) A(y, h) \frac{d x d y d h}{h} ; f, A \in C_{0}^{\infty} .
\end{aligned}
$$

We shall then compose $L, L_{k}, \widetilde{L}_{N}$ with the convolution operator $R$ where the notation used is for instance

$$
R_{2^{k}} L(A)(x)=\int 2^{-n k} R\left(\frac{x-y}{2^{k}}\right) L A(y) d y .
$$

With this notation and denoting by $I$ the identity operator (i.e. convolution with $\delta$ ) we can write

$$
\begin{gathered}
L_{k}=-R_{2^{k}} \widetilde{L}_{2^{k}}+\left(I-R_{2^{k}}\right) L_{k}+R_{2^{k}} L=M_{k}^{(1)}+M_{k}^{(2)}+M_{k}^{(3)}, \\
L^{* *}=\sup _{k}\left|L_{k}\right| \leq \sum_{i=1}^{3} \sup _{k}\left|M_{k}^{(i)}\right|=\sum M^{(i)},
\end{gathered}
$$

and the three components of the above decomposition will be examined separately. We have $M^{(3)} \leq M L$ for the Hardy-Littlewood maximal function $M$. It follows that the $D_{p} \mapsto L_{p}$ norm of $M^{(3)}(p>1)$ is dominated by the corresponding norm of $L$ in $[7,8] . M^{(1)}$ will be analyzed in Section 1.2 below.

To deal with $M^{(2)}$ we shall introduce $\mathcal{L} A(x) \in \ell^{2},\left(A \in C_{0}^{\infty}\left(\mathbb{R}_{+}^{n+1}\right), x \in \mathbb{R}^{n}\right)$ a vector valued operator defined by

$$
\begin{gathered}
(\mathcal{L} A, f)=\sum_{\substack{k=-\infty \\
f_{j} \in C_{0}^{\infty}, f_{j}=0}}^{+\infty}\left(\left(I-R_{2^{k}}\right) L_{k} A, f_{k}\right) ; f=\left(\cdots f_{-1}, f_{0}, \cdots\right), \\
\text { for }|j| \text { large enough. }
\end{gathered}
$$

We shall then prove the

Proposition 1.1. Let the notation be as above and let $Q, a_{j}$ be as in Proposition 0.2 and let us assume that in (0.7) $F=F_{0}=c_{0}+\sum c_{j} z_{j}$ is affine. For all $1<p<\infty$ we then have:

$$
\|\mathcal{L} A\|_{L^{p}\left(\ell^{2}\right)} \leq C\|\Omega\|_{r}\left\|F_{0}\right\|\|A\|_{D_{p}} ; A \in C_{0}^{\infty},
$$

with $C=C(n, r, k, N, p)$ and

$$
\left\|F_{0}\right\|=\sum\left|c_{j}\right| ;\|f\|_{L^{p}\left(\ell^{2}\right)}^{p}=\int\left(\sum_{-\infty}^{+\infty}\left|f_{k}(x)\right|^{2}\right)^{p / 2} d x .
$$

In the next few lines I shall assume Proposition 1.1 and complete the proof of Proposition 0.2 . 


\subsection{The pointwise estimate for $M^{(1)}$}

We shall use the notation and the facts from [7, Section 2.1, 2.2]. We denote by $\widetilde{A}=A^{*}+S(h \nabla A)$ for $A \in C_{0}^{\infty}\left(\mathbb{R}_{+}^{n+1}\right)$ and we shall decompose $A=A_{1}+A_{2}$ so that

$$
\begin{gathered}
\operatorname{supp} A_{1} \subset\left[|x|+h \leq C_{1}\right], \operatorname{supp} A_{2} \subset\left[|x|+h \geq C_{2}\right] ; \\
\left\|A_{1}\right\|_{D_{1}} \leq C\|\widetilde{A}\|_{L^{1}\left(|x| \leq C_{3}\right)} \leq C M \widetilde{A}(0),
\end{gathered}
$$

where $C_{i}$ are appropriate constants and $M$ will denote throughout the HardyLittlewood function. We shall use (1.8) to deduce

$$
\left|R \widetilde{L}_{1} A(0)\right| \leq C M \widetilde{A}(0)+C M M_{\Omega} A^{*}(0),
$$

where (cf. [6, II, Section 4])

$$
M_{\Omega} f(x)=\sup _{r} r^{-n} \int_{|x-y| \leq r}|f(x-y) \bar{\Omega}(y /|y|)| d y ; \bar{\Omega}(\sigma)=|\Omega(\sigma)|+1, \sigma \in \Sigma .
$$

Once (1.9) has been proved, by scaling and translation we obtain the pointwise control of $R_{N} \widetilde{L}_{N},(N \geq 0)$ and of $M^{(1)}$ by $M \widetilde{A}(x)+M M_{\Omega} A^{*}(x)$ as required.

Proof of (1.9). With $C>0$ and $C_{2}$ large enough we have $\widetilde{L}_{1} A_{2}(x)=0$ for $|x| \leq$ $C$, and therefore

$$
\widetilde{L}_{1} A(x)=\widetilde{L}_{1} A_{1}(x)=L A_{1}(x)-L_{0} A_{1}(x) ;|x| \leq C .
$$

But by (1.8) and the compactness of the support of $Q(x)$ in (0.7) we have $\left|L_{0} A_{1}(x)\right| \leq C M_{\Omega} A^{*}(x),|x| \leq C$. From the main Theorem of [7] and (1.8) we have on the other hand

$$
\left\|L A_{1}\right\|_{1} \leq C\left\|A_{1}\right\|_{D_{1}} \leq C\left\|\widetilde{A}_{1}\right\|_{1} \leq C M \widetilde{A}(0) .
$$

These two estimates are inserted in (1.11) and then we convolve with $R$. (1.9) follows.

\subsection{Proof of Proposition 0.2}

\section{Reduction to the discrete parameter}

Let $Q$ be as in (0.7) we then have

$$
\begin{gathered}
\int_{2^{k} \leq h \leq 2^{k+1}}|Q(x, y ; h) A(y, h)| \frac{d y d h}{h} \leq C M_{\Omega} A^{*}(x) ; \\
k \in \mathbb{Z}, x \in \mathbb{R}^{n}, A \in C_{0}^{\infty}\left(\mathbb{R}^{n+1}\right),
\end{gathered}
$$

with the notation (1.10). This implies that for the Proposition 0.2 it suffices to prove (0.9) with $L^{*}$ replaced by the $L^{* *}$ of (1.4). From the above, and Proposition 1.1, it follows therefore that Proposition 0.2 holds for affine functions $F=F_{0}$. 


\section{Reduction to the affine function}

Once the Proposition 0.2 has been proved for an affine function $F_{0}$ and $p \geq p_{0}$ large enough then this proposition holds for all $F \in C^{N}$ and all $1<p<\infty$.

The proof of this reduction is not trivial but it follows the same strategy as the reduction in [7, Section 7]. For the convenience of the reader I shall present this reduction in an essentially self contained manner in Section 3 below. $A_{1}$-weights, Carleson measures, and non trivial facts from $[5,7]$ will be used in that reduction.

\section{Proof of Proposition 1.1}

\subsection{The $L^{2}$-estimates}

Here $R$ will be as in Section 1 but $Q$ will be, a priori, an arbitrary $L^{1}\left(\mathbb{R}^{n}\right)$ function. We shall use furthermore the following notation

$$
\begin{aligned}
Q_{h}^{k} & =\left(I-R_{2^{k}}\right) Q_{h} \mathbf{I}\left(h>2^{k}\right), \\
B(f, A) & =\sum_{k=-\infty}^{+\infty} \int f_{k}(x) Q_{h}^{k}(x-y) A(y, h) \frac{d y d x d h}{h} ; A, f \text { as in (1.5), }
\end{aligned}
$$

where I denotes the indicator function. We shall recall the notation $\|A\|_{T_{2}^{2}}^{2}=$ $\int|A(y, h)|^{2} \frac{d y d h}{h}$ from [2] and we have:

\section{Lemma 2.1.}

$$
|B(f, A)| \leq C\|f\|_{L^{2}\left(\ell^{2}\right)}\|A\|_{D_{2}} .
$$

If the Fourier transform satisfies $\widehat{Q}(\xi)=O\left(|\xi|^{-\delta}\right)$ for some $\delta>0$ we have:

$$
|B(f, A)| \leq C\|f\|_{L^{2}\left(\ell^{2}\right)}\|A\|_{T_{2}^{2}} .
$$

We shall denote by $\widehat{A}(\xi, h)$ the partial Fourier transform of $A(\cdot, h)$ and denote by $S(\xi)=|1-\widehat{R}(\xi)|^{2}, T(\xi)=|\xi|^{-1}|\widehat{Q}(\xi)|^{2}$ and

$$
\|Q\| \|^{2}=\sup _{\xi} \sum_{k} \int \frac{\left|\widehat{Q}_{h}^{k}(\xi)\right|^{2}}{h|\xi|} \frac{d h}{h}=\sup _{\xi} \sum_{k} \int_{h>2^{k}} S\left(2^{k} \xi\right) T(h \xi) \frac{d h}{h} .
$$

By Plancherel and Hölder we have then

$$
\begin{aligned}
|B(f, A)|^{2} & \leq\|f\|_{L^{2}\left(\ell^{2}\right)}^{2} \sum_{k} \int\left(\int\left|\widehat{Q}_{h}^{k}(\xi) \widehat{A}(\xi, h)\right| \frac{d h}{h}\right)^{2} d \xi \\
& \leq\|f\|_{2}^{2} \int\left(\sum_{k} \int_{0}^{\infty} \frac{\left|\widehat{Q}_{h}^{k}(\xi)\right|^{2}}{h|\xi|} \frac{d h}{h}\right)\left(\int h|\xi||\widehat{A}(\xi, h)|^{2} \frac{d h}{h}\right) d \xi,
\end{aligned}
$$


(with the notation (1.7)). By partial integration (as in [7, Section 2.9]) on the last factor of the right hand side of (2.5) we see that (2.2) is a consequence of the fact that $\left\||Q \||<+\infty\right.$. This fact is easy to verify: We shall replace $S(\xi)$ by $|\xi|^{a} \wedge 1$ for some (arbitrary $c f$. Section 1$) a>1$ and replace $T(\xi)$ by $|\xi|^{-1}$. After this substitution we shall replace the discrete summation in the definition (2.4) by the continuous integral so that

$$
\left\||Q \||^{2} \leq C \sup _{\xi} \iint_{h>\ell}\left(|\ell \xi|^{a} \wedge 1\right)|h \xi|^{-1} \frac{d \ell}{\ell} \frac{d h}{h} .\right.
$$

This is useful because it makes the scaling $|\xi| h=h^{\prime},|\xi| \ell=\ell^{\prime}$ obvious and so, in proving that ||$Q|\||<+\infty$, we can just take $|\xi|=1$. We are thus just left with:

$$
\sum_{k<j}\left(2^{a k} \wedge 1\right) 2^{-j} \approx \sum_{j \leq 0} 2^{(a-1) j}+\sum_{j \geq 0} j 2^{-j} \leq C .
$$

We have in fact proved the more precise version of the lemma

$$
|B(f, A)| \leq C \mid\|Q\|\|\|\left\|_{2}\right\| A \|_{D_{2}} .
$$

For the estimate (2.3) we shall use Plancherel again and what has to be seen is that for each fixed $\xi$ (but uniformly in $\xi$ ) the matrix $\left(a_{k}(h)=\widehat{Q}_{h}^{k}(\xi) ; k \in \mathbb{Z}, h>0\right)$ defines an $\ell^{2} \rightarrow L^{2}\left(\frac{d h}{h}\right)$ bounded operator. We have $\left|a_{k}(h)\right|=S\left(2^{k} \xi\right)|\widehat{Q}(h \xi)| \mathbf{I}\left[2^{k}<\right.$ $h$ ] and by Schur's Lemma this operator norm can be estimated by

$$
\sup _{k} \int_{0}^{\infty}\left|a_{k}(h)\right| \frac{d h}{h}+\sup _{h} \sum_{k}\left|a_{k}(h)\right| \text {. }
$$

Here we shall proceed as before and replace $S(\xi)$ by $|\xi|^{a} \wedge 1$ and $|\widehat{Q}(\xi)|$ by $1 \wedge|\xi|^{-\delta}$ and then use the analogue of the continuous time integration for (2.8) as in (2.6). This allows us to scale again and assume that $|\xi|=1$. In (2.8) we are thus left with

$$
\begin{aligned}
& \sup _{k}\left(2^{a k} \wedge 1\right) \sum_{j>k}\left(2^{-\delta j} \wedge 1\right) \leq \sup _{k}\left(2^{a k} \wedge 1\right)\left(2^{-\delta k} \wedge(|k|+1)\right), \\
& \sup _{j}\left(2^{-\delta j} \wedge 1\right) \sum_{k<j}\left(2^{a k} \wedge 1\right) \leq \sup _{j}\left(2^{-\delta j} \wedge 1\right)\left(2^{a j} \wedge(|j|+1)\right),
\end{aligned}
$$

and both the above are $<+\infty$.

\section{Remark 2.2.}

(i) If we use the space variable $x$, the convolution kernel of the above $\ell^{2} \mapsto L^{2}\left(\frac{d h}{h}\right)$ operator is given by the matrix $b_{k}(h)=\left(Q_{h}(x)-R_{2^{k}} Q_{h}(x)\right) \mathbf{I}\left(h>2^{k}\right)$. For fixed $x$ this is a $2 \rightarrow 2$ bounded operator provided that $Q$ is smooth. This is seen by Hardy's inequality and it gives the size estimate for the generalized T1 theorem of Section 0.3. We shall not pursue the matter further but an alternative proof of (2.3) can be given in these lines. 
(ii) The condition $O\left(|\xi|^{-\delta}\right)$ on the Fourier transform is verified for the kernel $Q(x)=\Omega\left(\frac{x}{|x|}\right) \theta(|x|)$ of (0.7) cf. [4], [6, VIII 5.22].

\subsection{The scaled kernel}

We shall here scale $Q$ and consider $Q_{s}=s^{-n} Q\left(\frac{x}{s}\right)(0<s<1)$ and we shall also assume here that $\widehat{Q}(\xi)=O\left(|\xi|^{\delta}\right)$ for some $0<\delta \leq 10^{-10}$. In terms of the integral (2.6) we can then estimate

$$
\begin{aligned}
\left.\left\|Q_{s}\right\|\right|^{2} & \leq C \iint_{\ell<h} \frac{\left(\ell^{a} \wedge 1\right)\left((s h)^{2 \delta} \wedge 1\right)}{h} \frac{d h}{h} \frac{d \ell}{\ell} \\
& \leq C \int\left(h^{a} \wedge(|\log h|+1)\right)\left((s h)^{2 \delta} \wedge 1\right) \frac{d h}{h^{2}} \\
& \leq C s^{2 \delta} \int\left(h^{a} \wedge(|\log h|+1)\right) h^{2 \delta-2} d h=O\left(s^{2 \delta}\right) .
\end{aligned}
$$

Remark 2.3. In the applications that we have in mind in Section 2.4 (cf. (2.29)) it is not exactly the scaled $Q_{s}$ in (2.1) that will be used but the variant $Q * \check{Q}_{s}$ that is involved in:

$$
B_{3}^{0}(f, a)=\sum_{k=-\infty}^{\infty} \int \check{Q}_{s h} f_{k}(x) Q_{h}^{k}(x-y) A(y, h) \frac{d x d y d h}{h} .
$$

Proceeding as in (2.10) we see that $\|\left|Q * \check{Q}_{s}\right|||=O\left(s^{\delta}\right)$.

\section{The modified form}

We shall improve the estimate (2.3) for the modified form

$$
\begin{aligned}
B(f, A ; s)= & \sum_{k=-\infty}^{+\infty} \int_{s^{2} h>2^{k}} f_{k}(x) Q_{s h}^{k}(x-y) A(y, h) \frac{d y d x d h}{h} ; \\
& f, A \in C_{0}^{\infty}, 0<s<1 .
\end{aligned}
$$

We shall need the estimate

$$
|B(f, A ; s)| \leq C s^{\epsilon}\|f\|_{L^{2}}\|A\|_{T_{2}^{2}} ; 0<s<1,
$$

for some $\epsilon>0$. To see this we first reduce the corresponding Schur estimate to continuous time integration (as in (2.6)). We then use the scaling that allows us to normalize to $|\xi|=1$. We then make the additional change of variables $h \mapsto$ $s h, \ell \mapsto s \ell$ in that integration. Had we left the integration range in $\left[s h>2^{k}\right]$ the 
above transformations would not have changed the estimate (2.3). But the factor $s^{2}$ gives rise instead to a matrix

$$
M_{\sigma}=\left(\left(2^{a k} \wedge 1\right) \wedge\left(2^{-\delta j} \wedge 1\right) \mathbf{I}(k<j-\sigma)\right)_{k, j} ; \sigma=-\log s .
$$

As before the $\left\|M_{\sigma}\right\|_{2 \rightarrow 2}$ can be estimated by the analogue of (2.9):

$$
\begin{aligned}
& \sup _{k}\left(2^{a k} \wedge 1\right)\left(2^{-\delta(k+\sigma)} \wedge(|k|+\sigma+1)\right), \\
& \sup _{j}\left(2^{-\delta j} \wedge 1\right)\left(2^{a(j-\sigma)} \wedge(|j|+\sigma+1)\right) .
\end{aligned}
$$

(2.13) follows.

\subsection{Proof of Proposition 1.1 for $p=2$}

For an affine function $F_{0}$ and $Q$, as in Proposition 1.1, and $\check{P} \in C_{0}^{\infty}$ s.t. $\int \check{P}=1$, we define

$$
\begin{aligned}
B(f, A) & =\sum_{k=-\infty}^{+\infty} \int f_{k}(x) Q_{h}^{k}(x-y) F_{0}\left([a]_{x, y}\right) A(y, h) \frac{d x d y d h}{h}, \\
B_{0}(f, A) & =\sum_{k=-\infty}^{+\infty} \int f_{k}(x) Q_{h}^{k}(x-y) \check{P}_{h} a(y) A(y, h) \frac{d x d y d h}{h} .
\end{aligned}
$$

The estimate of the above $B$ with $F_{0} \equiv 1$ is given in (2.2) and this gives the proof of the Proposition 1.1 for $p=2$ and $F_{0} \equiv 1$. But the same estimate gives

$$
\left|B_{0}(f, A)\right| \leq C\|f\|_{L^{2}\left(\ell^{2}\right)}\|A\|_{D_{2}}\|a\|_{\infty} .
$$

This is because $\check{P}_{h} a(y) \in D_{\infty}$ and therefore this factor can be absorbed with $A(y, h)($ Cf. [7, Section 2.2, 7] also [2]).

For the Proposition 1.1 we have to prove the estimate (2.17) for the form $B$ in (2.16). From the above it follows that it suffices to prove the same estimate for the correcting form:

$$
\begin{aligned}
B_{1}(f, A)= & \sum_{\substack{k=-\infty \\
a \in C_{0}^{\infty},\|a\|_{\infty} \leq 1 .}}^{+\infty} f_{k}(x) Q_{h}^{k}(x-y)\left([a]_{x, y}-\check{P}_{h} a(y)\right) A(y, h) \frac{d y d x d h}{h} ; \\
&
\end{aligned}
$$

Towards that we shall modify $B_{1}$ further and define first $B_{2}(f, A)$ where in the integrand of (2.18) we smooth out and replace $f_{k}(x)$ by $\check{P}_{h} f_{k}(x)$ (with $\int \check{P}=1$ and $\left.I-\check{P}_{h}=\int_{0}^{1} \check{Q}_{s h} \frac{d s}{s}\right)$. Another modification of $B_{1}$ is $B_{3}(f, A ; s), 0<s<1$, where 
we replace $f_{k}$ by $\check{Q}_{s h} f_{k}(x)$ and where in addition the summation in $k$ is restricted to the range $\left[2^{k} \leq s^{2} h\right]$. Finally we consider $B_{4}(f, A ; s)$ with the same $\check{Q}_{s h} f_{k}(x)$ in the integrand but where the summation of the $k$ 's extend to the complementary range $s^{2} h<2^{k} \leq h$.

We obtain thus the decomposition

$$
\begin{aligned}
B_{1}(f, A) & =B_{2}(f, A)+\int_{0}^{1} B_{3}(f, A ; s) \frac{d s}{s}+\int_{0}^{1} B_{4}(f, A ; s) \frac{d s}{s} \\
& =X+Y+Z
\end{aligned}
$$

and we shall estimate the three components separately.

\section{Estimate of $X$}

We assume that $f \in L^{2}\left(\ell^{2}\right), A \in D_{2}$ and we have:

$$
\begin{aligned}
& X=\int U(x, h) V(x, h) \frac{d x d h}{h} ; \\
& U(x, h)=\sum_{2^{k} \leq h} \check{P}_{h}\left(I-R_{2^{k}}\right) f_{k} \in T_{2}^{2}(c f .(2.3)) \\
& V(x, h)=\int Q_{h}(x-y)\left([a]_{x, y}-\check{P}_{h} a(y)\right) A(y, h) d y \\
&|V| \leq\left(\int\left|Q_{h}(x-y)\right|\left|[a]_{x, y}-\check{P}_{h} a(y)\right|^{2} d y\right)^{1 / 2}\left(\int\left|Q_{h}(x-y) A^{2}(y, h)\right| d y\right)^{1 / 2} \\
&=V_{1} V_{2} .
\end{aligned}
$$

With the notation of [2] we have $V_{2} \in T_{\infty}^{2} c f$. [7, Section 4.1] (i.e. the non tangential maximal function lies in $L_{2}$ ), $V_{1} \in T_{2}^{\infty} c f$. [7, Section 3.4] (i.e. Carleson measures as in [2]). Observe here that because of the compactness of the support of $Q$ the localization in [7, (3.12), (3.13)] is symmetric in $x$ and $y$. Therefore it does not matter that in [7, (3.4)] we define $\beta^{2}(x, h)$ with $\check{P}_{h} a(y)$ instead of $\check{P}_{h} a(x)$ and we still have the Carleson condition for $\left(\beta^{2}(x, h) \frac{d x d h}{h}\right)$. Now it follows from [2] that $V \in T_{\infty}^{2} T_{2}^{\infty} \subset T_{2}^{2}$ and this together with the expression of $X$ in (2.20) gives the required estimate for $X$. More explicitly we can use the trivial fact that $T_{2}^{2} T_{\infty}^{2} \subset T_{2}^{1}$ and the (non trivial) duality between $T_{2}^{1}$ and $T_{2}^{\infty}$ (as in [7, (4.13)]) to obtain the required

$$
|X| \leq C\|f\|_{2}\|A\|_{D_{2}} .
$$




\section{Estimates of $Y$}

For $B_{2}$ we have exactly the same scalar product as in (2.20) but now $U$ is replaced by $\int_{0}^{1} U_{s} \frac{d s}{s}$ where

$$
U_{s}(x, h)=\sum_{2^{k} \leq s^{2} h} \check{Q}_{s h}\left(I-R_{2^{k}}\right) f_{k} ;\left\|U_{s}\right\|_{T_{2}^{2}} \leq C s^{\epsilon}\|f\|_{L^{2}\left(\ell^{2}\right)},
$$

because of (2.13). The same argument as for (2.22) and integration in $s$ gives therefore:

$$
\left|B_{3}(f, A ; s)\right| \leq C s^{\epsilon}\|f\|_{L^{2}\left(\ell^{2}\right)}\|A\|_{D_{2}} ;|Y| \leq C\|f\|_{L^{2}\left(\ell^{2}\right)}\|A\|_{D_{2}} .
$$

\section{Estimates of $Z$}

This is deeper. The summation of the $k$ 's that gives $B_{4}$ consists of $c|\log s|+c$ terms and the required estimate follows from the fact that each of these terms is

$$
O\left(s^{\epsilon}\|f\|_{L^{2}\left(\ell^{2}\right)}\|A\|_{D_{2}}\right) ; \text { some } \varepsilon>0,
$$

summing and integrating in $s$ as in (2.19) gives the required estimate and completes the proof of Proposition 1.1.

The estimate (2.25) is not obvious. It is in fact the key estimate of [7, (1.32)] and the reader will have to read a good part of [7] to understand how this is proved. (The additional factor $\left(I-R_{2^{k}}\right)$ gives no problem here because it can be absorbed with $f_{k}$, and the additional term $\check{P}_{h} a(y) \in D_{\infty}$ is absorbed with $A(y, h)$ as before.) This estimate (1.32) is on the other hand stated in the introduction of [7]. A quick glance at that introduction could perhaps satisfy the less demanding readers.

\subsection{Proof for $1<p<\infty$}

Having proved that $\mathcal{L}: D_{p} \rightarrow L^{p}\left(\ell^{2}\right)$ is bounded for $p=2$, it follows that the same thing holds for $0<p \leq 1$. Here as in [7, Section 2.4, 2.5] it suffices to test on the atoms of $D_{p}$. Observe however that here we cannot reduce the test to unit atoms only, because a priori we cannot scale. But this makes no difference. On the other hand the operator $\mathcal{L}$ is local in the sense that $\operatorname{supp}(\mathcal{L} A) \subset \widetilde{I}$ for all $A$ with supp $A \subset T(I)\left(I \subset \mathbb{R}^{n}\right.$ is a cube, $\tilde{I}$ is the concentric cube $c$-times as large and $T(I)$ is the tent above $I[2,6,7]$. Cf. also (3.3) below). It follows that for an atom $A \in D_{p}$ we do not have to worry about $\mathcal{L} A$ far out. Therefore no size estimate for $Q$ is needed here.

Having proved the boundedness for $\mathcal{L}$ for $p \leq 1$ and $p=2$ we can interpolate and obtain all the values $0<p \leq 2$ ( $c f$. [7, Section 2.7, A.3.2])

As in [7], in the scalar case, the values $2<p<\infty$ are much harder to handle and in particular we shall need to use concrete size and smoothness estimates for the 
kernel of $\mathcal{L}$ as $[7$, Section $2.5,2.6]$. We shall analyze these estimates again in more detail in the Appendix 2 below. Here I shall assume the results of the Appendix and complete the proof of the boundedness of $\mathcal{L}$ for $2 \leq p<\infty$. I shall use the forms $B_{i}, 0 \leq i \leq 4$ of Section 2.3 and (as in [7, (6.16)]) I shall first prove estimates for the Hardy space:

$$
\left|B_{i}(f, A)\right| \leq C_{i}\|f\|_{H^{1}\left(\ell^{2}\right)}\|A\|_{D_{\infty}} .
$$

For $i=2$ the form $B_{2}$ is smooth in the sense of [7, Section 2.6]. (Cf. Lemma (i) of the Appendix 2.) For $C_{2}$ we can therefore take a constant $C$. The $C_{3}, C_{4}=C(s)$ will on the other hand depend on $s$ but as in [7, Section 2.7] ( $c f$. Lemma (ii) of the Appendix 2) we can assert that for any $\lambda>0$ we have $C_{3}, C_{4} \leq C_{\lambda} s^{-\lambda}, 0<s<1$. The estimate (2.26) for $i=3,4$ can thus be interpolated with (2.24), (2.25). (This is the Lemma (iii) of the Appendix 2) and we deduce that

$$
\left|B_{i}(f, A ; s)\right| \leq C s^{\epsilon_{p}}\|f\|_{L^{q}\left(\ell^{2}\right)}\|A\|_{D_{p}} ; i=3,4,2<p<\infty, 1 / p+1 / q=1,
$$

where $C$ and $\epsilon_{p}>0$ depend on $p$. The above inserted in (2.19) gives

$$
\left|B_{i}(f, A)\right| \leq C\|f\|_{L^{q}\left(\ell^{2}\right)}\|A\|_{D_{p}} ; i=1,1 / p+1 / q=1,2 \leq p<\infty .
$$

Now I shall examine $B_{0}$ in (2.16) and decompose it

$$
B_{0}(f, A)=B_{2}^{0}(f, A)+\int_{0}^{1} B_{3}^{0}(f, A ; s) \frac{d s}{s},
$$

where $B_{2}^{0}(f, A)$ is obtained from $B_{0}(f, A)$ by replacing in the integrand $f_{k}$ by $\check{P}_{h} f_{k}$, and $B_{3}^{0}$ is obtained by replacing in the integrand $f_{k}$ by $\check{Q}_{s h} f_{k}$. This is analogous to what we did in Section 2.3 for the decomposition (2.19) of $B_{1}$ (except that now we do not need to modify the summation range of $k$ and we do not need to define the analogue of $B_{4}$ ). By the smoothness properties of these forms ( $c f$. Appendix 2) we obtain as before

$$
\left|B_{i}^{0}(f, A)\right| \leq C_{i}\|f\|_{H^{1}\left(\ell^{2}\right)}\|A\|_{D_{\infty}} ; i=2,3,
$$

with $C_{2}=C$ is a constant and $C_{3} \leq C_{\lambda} s^{-\lambda}$ for any $\lambda>0$. The estimate (2.30) can then be interpolated with $(2.2)^{\prime},(2.10),(2.11)$ and we obtain ( $c f$. Appendix 3)

$$
B_{i}^{0}(f, A) \leq C_{i}\|f\|_{L^{q}\left(\ell^{2}\right)}\|A\|_{D_{p}} ; 2 \leq p<\infty, 1 / p+1 / q=1,
$$

with $C_{2}=C$ and $C_{3} \leq C_{\epsilon} s^{\epsilon}$ (for some $\epsilon>0$ ). If we insert (2.31) in (2.29) we obtain a proof of (2.28) for $i=0$. Since $B=B_{0}+B_{1}$ we have the proof of the Proposition 1.1 for $2 \leq p<+\infty$. 


\section{Reduction of Proposition 0.2 to affine functions}

One says (cf. [6]) that $0 \leq \sigma \in A_{\infty}$ if there exists $r>1, C>0$ such that

$$
\left(\frac{1}{|I|} \int_{I} \sigma^{r} d x\right)^{1 / r} \leq C \frac{1}{|I|} \int_{I} \sigma d x ; \text { I cube of } \mathbb{R}^{n},
$$

were $|I|$ denotes the Lebesgue measure of $I$. For the Properties 3.1 and 3.2 below the only thing that counts is this: For each $0<\epsilon<1$ and each $g \in L_{\text {loc }}^{1}$ such that the Hardy-Littlewood maximal function $M g$ is not identically $+\infty$ we have $(M g)^{\epsilon} \in A_{\infty}$. (We even have $(M g)^{\epsilon} \in A_{1} \subset A_{\infty}$ but this here can be ignored).

We shall consider here sublinear local mappings $L$ from functions $A$ on $\mathbb{R}_{+}^{n+1}$ to $L(A)$ a function on $\mathbb{R}^{n}$. To wit:

$$
|L(\lambda A)|=|\lambda||L A| ; \lambda \in \mathbb{R},\left|L\left(A_{1}+A_{2}\right)\right| \leq\left|L A_{1}\right|+\left|L A_{2}\right| .
$$

There exists $C_{0}>0$ such that for all cube $I \subset \mathbb{R}^{n}$ we have:

$$
\begin{aligned}
\operatorname{supp} A & \subset T(I)=\left[(x, h) ; h<c \operatorname{dist}\left(x, I^{c}\right)\right] \Rightarrow \\
\Rightarrow \operatorname{supp} L A & \subset C_{0} I=\text { the concentric cube } C_{0}-\text { times as large. }
\end{aligned}
$$

Property 3.1. Let us assume that $L$ satisfies (3.2) and that for all $\sigma \in A_{1}$ the mapping

$$
L: D_{1}(\sigma) \longrightarrow L_{1}(\sigma)
$$

is bounded. Then

$$
L: D_{p} \longrightarrow L_{p}
$$

bounded for all $1 \leq p<+\infty$.

Here $A \in D_{1}(\sigma)$ means that $\widetilde{A}=A^{*}+S(h \nabla A) \in L_{1}(\sigma)(c f$. [7, A.1] for more details on these weighted $D_{p}$-spaces). The above property is an immediate consequence of the property of $A_{1}$ weights that I stated above. $C f$. [7, Section 2.8] for a two line proof of Property 3.1. Here we shall need to use this property for the vector valued spaces $L^{p}\left(\ell^{2}\right)$. The prototype of the Property 3.1 can be found in [5].

Property 3.2. Let us assume that L satisfies (3.2) (3.3) let us assume that (3.5) bounded for all $1 \leq p<+\infty$ then (3.4) is bounded for all $\sigma \in A_{\infty}$.

Once more the $L_{p}$-spaces are here vector valued. The local property (3.3) is now essential because we use here the atomic decomposition of $D_{1}(\sigma)(c f$. [7, Section 2.8 and A.3.5.])

We shall consider now $\sigma \in A_{\infty}$ and $Q_{h}(x-y)=\Omega\left(\frac{x-y}{|x-y|}\right) \theta_{h}(|x-y|)$ as in (0.7) with $\Omega \in L^{r}(\Sigma)(r>1)$ and $\theta \in C_{0}^{\infty}$. We shall assume that $\int \check{P}=1$ and define as in $[7,(3.15)]$

$$
\beta_{\sigma}^{2}(x, h)=\int\left|Q_{h}(x-y)\right|\left|[a]_{x, y}-\check{P}_{h} a(x)\right|^{2} \sigma(y) d y .
$$




\section{Property 3.3.}

$$
\left|\int \beta_{\sigma}^{2}(x, h) A(x, h) \frac{d x d h}{h}\right| \leq C\|a\|_{\infty}^{2} \int A^{*}(x) \sigma(x) d x ; A \geq 0 .
$$

where here $A^{*}(x)$ denotes the non tangential maximal function $\sup [|A(z)| ; z \in$ $\left.\Gamma_{\delta}(x)\right]$.

It is easy to see that the right hand side of (3.7) is essentially independent of the apature of the wedge $\delta>0$ (cf. [7, A.1]). The proof of (3.7) is given in the Appendix 1.

\section{Proof of the reduction of Section 1.3}

We follow closely the strategy of [7, Section 7]. Let

$$
L_{X} A(x)=\sup _{\epsilon, M}\left|\int_{\epsilon<h<M} Q_{h}(x-y) X(x, y ; h) A(y, h) \frac{d y d h}{h}\right| .
$$

When $X(x, y ; h)=F_{0}\left([a]_{x, y}\right)=F_{0}$ for an affine $F_{0}=c_{0}+\Sigma_{i} c_{i} z_{i}$ I shall denote (3.8) by $L_{0}$. By Proposition 1.1 and the above Property 3.2 we deduce the boundedness of

$$
L_{0}: D_{1}(\sigma) \longrightarrow L_{1}(\sigma)\left(: \ell^{2} \text { valued } L^{2}\right),
$$

for $\sigma \in A_{\infty}$. (3.9) also holds if in (3.8) we set $X=\Phi\left(\check{P}_{h} a(y)\right)=\Phi$ for some $\Phi \in C^{N}$, because then $X$ is independent of $x$ and $X \in D_{\infty}$ and can therefore by absorbed with $A$ ( $c f$. [7, Section 2.2]). The Property 3.2 applies thus again. The same thing clearly also holds for a product $X=F_{0} \Phi$ as above. Let us consider the Taylor expansion of $F \in C^{N}$

$$
F\left([a]_{x, y}\right)=F_{0} \Phi+X(x, y ; h) ; X=O\left(\left|[a]_{x, y}-\check{P}_{h} a(y)\right|^{2}\right) ; a \in C_{0}^{\infty},
$$

where $F_{0}, \Phi$ are as above. We shall then apply Property 3.3 to $L_{X}(3.8)$ with the $X$ as (3.10). This will give the boundedness of:

$$
L_{X}: D_{1}(\sigma) \longrightarrow L_{1}(\sigma) ; \sigma \in A_{\infty} .
$$

To see this we use duality with $L^{\infty}$ (with respect to the weight $\sigma$ ) and use (3.7). But since $L_{X}$ is bounded in (3.11) for any $\sigma \in A_{\infty}$ if $X=F_{0} \Phi$, the boundedness of (3.11) with $X=F\left([a]_{x, y}\right)$ follows. The Property 3.1 completes the proof of the reduction.

\section{The Cotlar analysis - denouement}

In this paragraph and in the next I shall estimate the correcting term in the control of $(0.10)$. 


\subsection{Decomposition of the operators and notation}

\subsubsection{First set of notation}

All the $\check{P}$ will satisfy $\int \check{P}=1$ and we can write $I-\check{P}_{h}=\int_{0}^{1} \check{Q}_{s h} \frac{d s}{s}$ where $I$ denotes throughout the identity operator. Furthermore when confusion does not arise I shall identify functions with convolution operators. $R$ is as in Section $1, F \in C^{N}$ as in Section 0 and $Q(x)=\Omega\left(\frac{x}{|x|}\right) \theta(|x|)$ is as in (0.7). If $K=K(x, y)\left(x, y \in \mathbb{R}^{n}\right) \mathrm{I}$ shall denote by $\{K\}$ the operator whose kernel is $K(x, y)$. In the notation below a product of operators as above, denotes the composition of these operators.

The operators that give the correcting term in $(0.10)$ are

$$
T_{k}(h)=\int_{0}^{1}\left(I-R_{2^{k}}\right)\left\{Q_{h}(x-y) F\left([a]_{x, y}\right)\right\} \check{Q}_{s_{1}} h \frac{d s_{1}}{s_{1}}\left[h>2^{k}\right] ; k \in \mathbb{Z},
$$

with the abbreviation $\left[h>2^{k}\right]$ for the indicator function $\mathbf{I}\left(h>2^{k}\right)$ and where the $a$ 's are as in the Theorem. The constants in the estimates below depend on $n, r, k, N,\|F\|_{C^{N}}$ but not on the $a$ 's where we assume as before that $\|a\|_{\infty} \leq 1$. It is these operators that have to be analyzed.

\subsubsection{Decomposition of (4.1)}

We shall abbreviate and denote:

$$
\begin{aligned}
& \left\{Q_{h}(x-y) F\left([a]_{x, y}\right)\right\}=\{\cdots \mid h\} ; \\
& \left\{Q_{h}(x-y) F\left(\left[\check{P}_{h} a\right]_{x, y}\right)\right\}=\{1 \mid h\} ; \\
& \left\{Q_{h}(x-y) F\left(\left[\check{P}_{s h} a\right]_{x, y}\right)\left[\check{Q}_{s h} a\right]_{x, y}\right\}=\{2 \mid s, h\} .
\end{aligned}
$$

We have a first decomposition

$$
\{\cdots \mid h\}=\{1 \mid h\}+\int_{0}^{1}\{2 \mid s, h\} \frac{d s}{s},
$$

where $\{\cdots\}$ in the integrand is defined with the functions of $\nabla F$.

A further decomposition is obtained by inserting the decomposition of the identity $I=\check{P}_{h}+\left(I-\check{P}_{h}\right)$ :

$\left(I-R_{2^{k}}\right)\{\cdots \mid h\} \check{Q}_{s_{1} h}=\left(I-R_{2^{k}}\right) \check{P}_{h}\{\cdots \mid h\} \check{Q}_{s_{1} h}+\int_{0}^{1}\left(I-R_{2^{k}}\right) \check{Q}_{s_{2} h}\{\cdots \mid h\} \check{Q}_{s_{1} h} \frac{d s_{2}}{s_{2}}$.

If we combine (4.3) and (4.4) and multiply by $\left[h>2^{k}\right]$ we obtain a decomposition of $T_{k}(h)$ into four different operators. 


\subsubsection{An illustration and a further decomposition}

One of the above four components of $T_{k}(h)$ is

$$
\int_{0}^{1} \int_{0}^{1} \int_{0}^{1}\left(I-R_{2^{k}}\right) \check{Q}_{s_{1} h}\{2 \mid s, h\} \check{Q}_{s_{2}} h \frac{d s d s_{1} d s_{2}}{s s_{1} s_{2}}\left[h>2^{k}\right] .
$$

If we restrict the above integration to the range

$$
2^{-i}<s_{1}<2^{-i+1}, 2^{-j}<s_{2}<2^{-j+1}, 2^{-\ell}<s<2^{-\ell+1} ; 1 \leq i, j, \ell \in \mathbb{Z},
$$

we obtain operators $T_{k}(h \mid i, j, \ell)$ and a further decomposition of (4.5) into $\sum_{i, j, \ell} T_{k}(h \mid i, j, \ell)$.

Analogous, but simpler decompositions (that involve only one or two summation indices) can be given for the other three components of $T_{k}(h)$ in (4.3)-(4.4).

\subsubsection{The vector valued operator}

Let

$$
\mathbf{T}(h): L^{2} \longrightarrow L^{2}\left(\ell^{2}\right) ; \mathbf{T}(h) g=\left(\cdots T_{-1}(h) g, T_{0}(h) g, \cdots\right) .
$$

Observe that

$$
\mathbf{T}^{*}(h) \mathbf{T}\left(h^{\prime}\right)=\sum_{-\infty}^{+\infty} T_{k}^{*}(h) T_{k}\left(h^{\prime}\right) ; \mathbf{T}(h) \mathbf{T}^{*}\left(h^{\prime}\right)=\left(T_{k}(h) T_{r}^{*}\left(h^{\prime}\right)\right)_{k, r},
$$

where $(\cdot)_{k, r}$ indicates an operator entries (infinite) matrix that defines an operator in $\ell^{2}\left(L^{2}\right) \cong L^{2}\left(\ell^{2}\right)$.

\subsubsection{The h-integration}

For all the above operators we shall consider their integrated versions in $d h / h$ :

$$
T_{k}=\int_{0}^{\infty} T_{k}(h) \frac{d h}{h}, \mathbf{T}=\int_{0}^{\infty} \mathbf{T}(h) \frac{d h}{h}, \mathbf{T}_{i, j, \ell}=\int_{0}^{\infty} \mathbf{T}(h \mid i, j, \ell) \frac{d h}{h}, \cdots
$$

where $\mathbf{T}(h \mid i, j, \ell)$ is defined as in (4.6) from the operators $T_{k}(h \mid i, j, \ell)$.

\subsection{The Cotlar estimates}

The notation is as in Section 4.1. We shall prove that there exists $\epsilon>0$ and $\delta>0$ s.t.

$$
\begin{aligned}
& \left\|\mathbf{T}_{i, j, \ell}^{*}(h) \mathbf{T}_{i, j, \ell}\left(h^{\prime}\right)\right\|_{\mathrm{op}},\left\|\mathbf{T}_{i, j, \ell}(h) \mathbf{T}_{i, j, \ell}^{*}\left(h^{\prime}\right)\right\|_{\mathrm{op}} \\
& \leq C \exp (-\delta(i+j+\ell))\left(\left(h / h^{\prime}\right)^{\epsilon} \wedge\left(h^{\prime} / h\right)^{\epsilon}\right) ; i, j, \ell \geq 1 .
\end{aligned}
$$

Here \|\|$_{\text {op }}$ denotes the corresponding $L^{2} \longrightarrow L^{2}$ operator norm. Analogous estimates will be proved for the other components (that have one or two indices) of the decomposition Sections 4.1.2, 4.1.3. 


\section{The exponents $\epsilon$}

Throughout the $\epsilon>0$ that appears as an exponent will denote some positive constant that may differ from place to place.

From (4.8) Cotlar's Lemma applies ( $c f .[1,6])$ and we deduce:

$$
\|\mathbf{T}\|_{\mathrm{op}} \leq C ;\left\|\mathbf{T}_{i, j, \ell}\right\|_{\mathrm{op}} \leq C \exp (-\delta(i+j+\ell)),
$$

for some $\delta>0$.

The proof of (4.8) will be given below and is a straightforward adaptation of ideas of [7, Sections 8, 9] and of [1]. The only real difficulty in these proofs is to keep track of the notation which are unfortunately very involved.

\subsection{Estimate of $T^{*} T$}

The integrand of $\mathbf{T}_{i, j, \ell}^{*}(h) \mathbf{T}_{i, j, \ell}\left(h^{\prime}\right)$ that comes from (4.5) is:

$$
\check{Q}_{s_{1} h}\left\{2 \mid s_{2}, h\right\} \check{Q}_{s_{3} h}\left(I-R_{2^{k}}\right)^{2} \check{Q}_{s_{4} h^{\prime}}\left\{2 \mid s_{5}, h^{\prime}\right\} \check{Q}_{s_{6} h^{\prime}}\left[2^{k}<h \wedge h^{\prime}\right],
$$

where we integrate in the range:

$2^{-j}<s_{1}, s_{6}<2^{-j+1}, 2^{-i}<s_{3}, s_{4}<2^{-i+1}, 2^{-\ell}<s_{2}, s_{5}<2^{-\ell+1} ; i, j, \ell \geq 1$,

and sum in $k \in \mathbb{Z}$. The other components of the decomposition (4.3)-(4.4) give analogous (but simpler) expressions. If we integrate (4.10) in (4.11) we obtain $\mathbf{T}_{i, j, \ell}^{*}(h) \mathbf{T}_{i, j, \ell}\left(h^{\prime}\right)$. Finally in (4.10) and throughout, I abuse slightly the notation and use the same symbols $R,\{\cdots\}$ etc to indicate these operators and their adjoints. The functions that are used for these adjoints are of course different, but noting changes either in the notation or in the arguments that follow.

By taking adjoints if necessary we may assume that $h / h^{\prime}=e^{-a}, a \geq 0$. We shall denote $s_{i}=e^{-\sigma_{i}}\left(\sigma_{i} \geq 0\right)$ and use throughout the convention that in the exponentials that appear below e.g. $\exp \left(-\sigma_{1}\right), \exp \left(-\sigma_{3}+\sigma_{4}-a\right)$ etc. what is meant is $\exp \left(-c \sigma_{1}\right), \exp \left(-c \sigma_{3}+c \sigma_{4}-c a\right)$ etc. In other words the positive constants $c$ are omitted. Finally each of the factors in (4.10) has a bounded operator norm and the estimates given below are obtained by combining adjacent factors.

For the summation in $k$ in (4.10) two different ranges will be considered:

Range 1: $2^{k} \leq s_{3} h \wedge s_{4} h^{\prime}$

Range 2: (Z\ Range 1), and we shall use the fact that:

$$
\text { Cardinality of Range } 2 \leq c+c\left|\log s_{3}\right|+c\left|\log s_{4}\right| \text {. }
$$




\section{Estimate 1}

We shall estimate the $L^{1}$ norm of

$$
\sum_{k} \check{Q}_{s_{3} h}\left(I-R_{2^{k}}\right)^{2} \check{Q}_{s_{4} h^{\prime}}\left[2^{k}<h \wedge h^{\prime}\right]
$$

which is one of the segments of (4.10).

At this point it is instructive to consider first the other component coming from the first term of the right hand side of (4.4). The corresponding segment simplifies and is

$$
\sum_{2^{k}<h \wedge h^{\prime}} \check{P}_{h}\left(I-R_{2^{k}}\right)^{2} \check{P}_{h^{\prime}}=\sum_{2^{k}<h \wedge h^{\prime}} \check{Q}_{h}^{k} \check{Q}_{h^{\prime}}^{k} ;\left\|\check{Q}_{h}^{k}\right\|_{1}=O\left(2^{k} h^{-1}\right),
$$

because $\check{Q}_{h}^{k}=\left(I-R_{2^{k}}\right) \check{P}_{h}=\left(\left(I-R_{2^{k} h^{-1}}\right) \check{P}\right)_{h}$. The norm of each term, of (4.14) can also be estimated by $\left(h / h^{\prime}\right) \wedge\left(h^{\prime} / h\right)$ because of the cancellation. This gives

$$
\left\|\check{Q}_{h}^{k} \check{Q}_{h^{\prime}}^{k}\right\|_{1}^{2} \leq C\left[\left(h / h^{\prime}\right) \wedge\left(h^{\prime} / h\right)\right] 2^{k} h^{-1} .
$$

Summing up we see that we can estimate (4.14) by $\left(h / h^{\prime}\right)^{\varepsilon} \wedge\left(h^{\prime} / h\right)^{\varepsilon}=e^{-a}$.

For the original (4.13) exactly the same argument applies for the summation of $k \epsilon$ Range 1, and this gives therefore an estimate $\left(\frac{s_{3} h}{s_{4} h^{\prime}}\right)^{\mathcal{E}} \wedge\left(\frac{s_{4} h^{\prime}}{s_{3} h}\right)^{\varepsilon}$ for the sum of the $L^{1}$-norms. The same estimate holds for each individual term in the summation (4.13). In the range 2 we can thus use (4.12) to conclude

$$
(4.13) \leq C\left(1+\sigma_{3}\right)\left(1+\sigma_{4}\right) \exp \left(-\sigma_{3}+\sigma_{4}-a\right) .
$$

\section{Estimate 2}

Here we use [7, Section 8] (esp. (8.6) (8.7)) on each sub-product

$$
\left\{2 \mid s_{2}, h\right\} \check{Q}_{s_{3} h}\left(I-R_{2^{k}}\right) \text { or }\left(I-R_{2^{k}}\right) \check{Q}_{s_{4} h^{\prime}}\left\{2 \mid s_{5}, h^{\prime}\right\},
$$

in (4.10). Furthermore as before we can combine together the terms that come from $k \epsilon$ Range 1 . We obtain therefore for the $L^{2}$ operator norm of (4.10) the estimate

$$
\left(1+\left|\log s_{3}\right|+\left|\log s_{4}\right|\right)\left(\frac{s_{3}^{\epsilon}}{s_{2}^{\epsilon}} \wedge \frac{s_{4}^{\epsilon}}{s_{5}^{\epsilon}}\right) \sim\left(1+\sigma_{3}+\sigma_{4}\right)\left(\exp \left(-\sigma_{3}+\sigma_{2}\right) \wedge \exp \left(-\sigma_{4}+\sigma_{5}\right)\right),
$$

where the logarithmic factor comes from (4.12). We shall omit the details. The key observation however, as in (4.14), is that with $k \in \operatorname{Range} 1\left(I-R_{2^{k}}\right) \check{Q}_{s_{3} h}=$ $2^{k} s_{3}^{-1} h^{-1} \check{Q}_{s_{3} h}^{(k)}$ with the $\check{Q}^{(k)}$ 's staying in a "bounded set" of functions of $C_{0}^{\infty}$. The additional scalar factor comes about because the integral of $I-R_{r}$ is 0 and the diameter of its support is $r$. Here $r=2^{k} s_{3}^{-1} h^{-1}$. 
It will be instructive at this point to switch to the two components of the decomposition Section 4.1.2 that come from $\{1 \mid h\}$ in (4.3). I shall show how the above two estimates suffice to give the final control of these two components. Indeed the integrands in $\mathbf{T}^{*}(h) \mathbf{T}\left(h^{\prime}\right)$ that give these two components are respectively

$$
\begin{gathered}
\check{Q}_{s_{1} h}\{1 \mid h\} \check{Q}_{s_{3} h}\left(I-R_{2^{k}}\right)^{2} \check{Q}_{s_{4} h^{\prime}}\left\{1 \mid h^{\prime}\right\} \check{Q}_{s_{6} h^{\prime}}\left[2^{k}<h \wedge h^{\prime}\right], \\
\check{Q}_{s_{1} h}\{1 \mid h\} \check{P}_{h}\left(I-R_{2^{k}}\right)^{2} \check{P}_{h^{\prime}}\left\{1 \mid h^{\prime}\right\} \check{Q}_{s_{6} h^{\prime}}\left[2^{k}<h \wedge h^{\prime}\right] .
\end{gathered}
$$

For (4.19) if we use (4.16) on the middle three factors we obtain the estimate

$$
\exp \left(-\sigma_{3}+\sigma_{4}-a\right) \text {. }
$$

On the other hand in (4.19) from the four possible combinations of the type (4.17) (where now $s_{2}=s_{5}=1$ ) we obtain for the summation in Range 1the estimates

$$
s_{1}^{\epsilon} \wedge s_{3}^{\epsilon} \wedge s_{4}^{\epsilon} \wedge s_{6}^{\epsilon} .
$$

For this we observe that the summation of the middle three factors is also bounded by $C$ and thus the first (respectively last) two factors give $s_{1}^{\epsilon}$ (respectively $s_{6}^{\epsilon}$ ).

If we combine these with the logarithmic factor coming from the summation in $k$ in Range 2 (4.12) we finally obtain for (4.19) the estimate

$$
\left(1+\sigma_{3}\right)\left(1+\sigma_{4}\right)\left[e^{-\sigma_{1}} \wedge e^{-\sigma_{3}} \wedge e^{-\sigma_{4}} \wedge e^{-\sigma_{6}} \wedge \exp \left(-\sigma_{3}+\sigma_{4}-a\right)\right] .
$$

That this suffices to give (4.9) is easy to see [cf. (4.24) below].

An analogous, but simpler estimate can be obtained for (4.20). To treat the term (4.10) we shall need the following additional:

Estimate 3 [7, Section 8], [1, Section 5.3]

$$
\left\|\left\{2 \mid s_{2}, h\right\}\right\|_{\mathrm{op}} \leq C s_{2}^{\epsilon} ;\left\|\left\{2 \mid s_{5}, h^{\prime}\right\}\right\|_{\mathrm{op}} \leq C s_{5}^{\epsilon} .
$$

With this new estimate and with the previous treatment of (4.19) adapted now to the case $0<s_{2}, s_{5}<1$ we finally obtain for (4.10) the estimate

$$
\begin{aligned}
& \left(1+\sigma_{3}\right)\left(1+\sigma_{4}\right)\left[s_{2}^{\epsilon} \wedge s_{5}^{\epsilon} \wedge\left(\frac{s_{1}^{\epsilon}}{s_{2}^{\epsilon}} \wedge \frac{s_{3}^{\epsilon}}{s_{2}^{\epsilon}}\right) \wedge\left(\frac{s_{4}^{\epsilon}}{s_{5}^{\epsilon}} \wedge \frac{s_{6}^{\epsilon}}{s_{5}^{\epsilon}}\right) \wedge\left(\frac{s_{3} h}{s_{4} h^{\prime}}\right)^{\epsilon}\right] \\
& \approx\left(1+\sigma_{3}\right)\left(1+\sigma_{4}\right)\left[e^{-\sigma_{2}} \wedge e^{-\sigma_{5}} \wedge \exp \left(-\sigma_{1}+\sigma_{2}\right) \wedge \exp \left(-\sigma_{3}+\sigma_{2}\right)\right. \\
& \left.\wedge \exp \left(-\sigma_{4}+\sigma_{5}\right) \wedge \exp \left(-\sigma_{6}+\sigma_{5}\right) \wedge \exp \left(-\sigma_{3}+\sigma_{4}-a\right)\right] .
\end{aligned}
$$

To obtain the estimate (4.9) for the component (4.10), as in [7, Section 9] we apply on (4.23) the barrycenter inequality

$$
\operatorname{Min}\left[x_{1}, \cdots, x_{m}\right] \leq x_{1}^{\alpha_{1}} \cdots x_{m}^{\alpha_{m}} ; x_{j} \geq 0, \alpha_{j} \geq 0,(1 \leq j \leq m), \quad \sum \alpha_{j}=1,
$$

for an appropriate choice of the $\alpha_{j}$ 's, and where the $x_{j}$ are the exponentials of (4.23). We then integrate each $\sigma_{r}, 1 \leq r \leq 6$ in the corresponding interval $[i, i+$ $1],[j, j+1],[\ell, \ell+1]$ that is given by (4.11). (4.9) follows at once. 


\subsection{Estimate of $T T^{*}$}

The integrand of $\mathbf{T}_{i, j, \ell}(h) \mathbf{T}_{i, j, \ell}^{*}\left(h^{\prime}\right)$ that comes from (4.5) is

$$
\left(I-R_{2^{k}}\right) \check{Q}_{s_{1} h}\left\{2 \mid s_{2}, h\right\} \check{Q}_{s_{3} h} \check{Q}_{s_{4} h^{\prime}}\left\{2 \mid s_{5}, h^{\prime}\right\} \check{Q}_{s_{6} h^{\prime}}\left(I-R_{2^{r}}\right)\left[2^{k}<h\right]\left[2^{r}<h^{\prime}\right],
$$

where no summation in $k$ or $r$ is involved here and (4.25) represents the entry of the matrix $(\cdot)_{k, r}$ in (4.7). The other components of the decomposition (4.3)-(4.4) give analogous but simpler expressions.

If we integrate (4.25) in the range (4.11) we obtain

$$
A_{k, r}=T_{k}(h \mid i, j, \ell) T_{r}^{*}\left(h^{\prime} \mid i, j, \ell\right),
$$

and we have

$$
\left\|\mathbf{T}_{i, j, \ell}(h) \mathbf{T}_{i, j, \ell}^{*}\left(h^{\prime}\right)\right\|_{\mathrm{op}} \leq \sum_{k, r}\left\|A_{k, r}\right\|_{\mathrm{op}} .
$$

We shall use the same method and the same notation as in Section 4.3 to estimate (4.27). As in Section 4.3 we distinguish in the summation (4.27) the four ranges obtained from the decompositions

$$
\left[2^{k} \leq s_{1} h\right] \cup\left[s_{1} h \leq 2^{k}<h\right] ;\left[2^{r} \leq s_{6} h^{\prime}\right] \cup\left[s_{6} h^{\prime} \leq 2^{r} \leq h^{\prime}\right] .
$$

We shall in particular use the estimate 1-2-3 of Section 4.3 to estimate various segments of the product of operators appearing in (4.25). We shall then use the ranges (4.28) to sum in $k, r$. We shall omit the details. The estimate obtained is as before (4.23). We finally integrate in the same range for the $\sigma_{r}(1 \leq r \leq 6)$.

\subsection{Denouement}

The estimate that will be used is summarized in the following:

Lemma 4.1. With our previous notation

$$
\int_{\epsilon}^{M} \mathbf{T}(h) \frac{d h}{h}: L^{p} \longrightarrow L^{p}\left(\ell^{2}\right) ; 1<p<\infty,
$$

is bounded uniformly in $\epsilon, M>0$.

In (4.9) I gave a proof of (4.29) for $p=2$. The general $1<p<\infty$ will be proved in Section 5 below. Here I shall assume the results of [7] and (4.29) and complete the proof of (0.10). This, as explained in Section 0.2, will finish the proof of the theorem. What is needed is the control of

$$
\begin{gathered}
f \mapsto \sup _{N}\left|\Lambda_{N} f\right| ; \Lambda_{N} f(x)=\iint_{h<N} Q_{h}(x-y) F\left([a]_{x, y}\right)\left(I-\check{P}_{h}\right) f(y) \frac{d y d h}{h} ; \\
0<N \leq \infty .
\end{gathered}
$$


A consequence of the lemma is that

$$
f \mapsto \sup _{k}\left|\left(I-R_{2^{k}}\right)\left(\Lambda_{\infty}-\Lambda_{2^{k}}\right) f(x)\right|,
$$

is $L^{p} \longrightarrow L^{p}$ bounded (because $\ell^{2} \subset \ell^{\infty}$ ).

We shall prove that

$$
f \mapsto \sup _{N}\left|R_{N} \Lambda_{N} f(x)\right|
$$

is $L^{p} \rightarrow L^{p}$ bounded. From (4.31) and (4.32) $f \mapsto \sup \left|\Lambda_{2^{k}} f\right|$ is seen to be $L^{p}$ bounded, therefore by the argument of Section 1.3 the ${ }^{k} L^{p}$ boundedness (4.30) follows. Observe also that the full thrust of [7] is used together with the fact that $\check{P}_{h} f(x) \in D_{p}$ for $f \in L^{p}$ to guarantee the $p \rightarrow p$ boundedness of $\Lambda_{N}$. This finishes the proof.

To prove (4.32) it suffices to show that

$$
\left|R_{N} \Lambda_{N} f(x)\right| \leq C\left(M|f|^{p}\right)^{1 / p}(x) ; 1<p<\infty,
$$

where $M$ is the Hardy-Littlewood maximal function. But by translation and dilation we can assume in (4.33) that $x=0, N=1$. But then (4.33) is a consequence of the fact that $\Lambda_{1}$ is $L^{p}-L^{p}$ bounded. Indeed if we localize $f_{c}=f \chi_{[|x|<c]}$ we have $R_{1} \Lambda_{1} f(0)=R_{1} \Lambda_{1} f_{c}(0)$ because of the compactness of the support of $Q$. It follows that

$$
\left|R_{1} \Lambda_{1} f(0)\right|=\left|R_{1} \Lambda_{1} f_{c}(0)\right| \leq C\left\|\Lambda_{1} f_{c}\right\|_{p} \leq C\left\|f_{c}\right\|_{p} \leq C\left(M|f|^{p}\right)^{1 / p}(0),
$$

as needed.

From (4.33) it follows that (4.32) is $L^{p} \longrightarrow L_{p, \infty}$ bounded $1<p<\infty$ i.e. the weak $p$-boundedness. Interpolation gives the corresponding $L^{p}$-boundedness.

\section{The Calderon-Zygmund estimates}

\subsection{Terminology and basic facts $[1,6]$}

Let $K(x, y) \in H=\ell^{2},\left(x, y \in \mathbb{R}^{n}\right)$ be a vector valued kernel. We say that $K$ satisfies the standard estimates if:

$$
\text { Size estimate: }|K(x, y)| \leq C_{1}|x-y|^{-n} \text {. }
$$

Gradient estimate: $|\nabla K(x, y)|=\left|\nabla_{x} K\right|+\left|\nabla_{y} K\right| \leq C_{2}|x-y|^{-n-1}$.

Let $T, T^{*}$ be operators $L^{2} \longrightarrow L^{2}(H)$ and $L^{2}(H) \longrightarrow L^{2}$ with $\|T\|_{2 \rightarrow 2}=C_{3}$ and let us assume that the kernel of $T$ satisfies the standard estimates. In terms of 
the constants $C_{1}, C_{2}, C_{3}$ and with the notation $L_{1, \infty}$ for the weak $L_{1}$ space (Lorentz notation), the "norm" of:

$$
T: L^{1} \longrightarrow L_{1, \infty}(H), T^{*}: L_{1}(H) \longrightarrow L_{1, \infty}
$$

can then be bounded by

$$
C_{\theta}\left(C_{3}+C_{1}^{1-\theta} C_{2}^{\theta}\right) ; 0<\theta<1,
$$

where $C_{\theta}$ only depends on $n$ and $\theta$. (Cf. [1,6] for this basic fact). Let us also consider the condition ( $c f$. [6, I, Section 5])

$$
\int_{|x-y|>2\left|x-x^{\prime}\right|}\left(\left|K(x, y)-K\left(x^{\prime}, y\right)\right|+\left|K(y, x)-K\left(y, x^{\prime}\right)\right|\right) d y \leq C_{4} ; x, x^{\prime} \in \mathbb{R}^{n} .
$$

We obviously have $C_{4} \leq C_{\theta} C_{1}^{1-\theta} C_{2}^{\theta}$, but we can also improve the above and we can estimate the norms of (5.1) by

$$
C\left(C_{3}+C_{4}\right) .
$$

\subsection{The scalar valued illustration [7, Section 9]}

With the notation of Section 4.1 I considered in [7] the $L^{2} \longrightarrow L^{2}$ operators

$$
T_{i, j, \ell}=\int_{0}^{\infty} \frac{d h}{h} \iiint \check{Q}_{s_{1} h}\{2 \mid s, h\} \check{Q}_{s_{2}} \frac{d s d s_{1} d s_{2}}{s s_{1} s_{2}}
$$

where the $s$-integration is taken in

$$
2^{-i} \leq s_{1} \leq 2^{-i+1}, 2^{-j} \leq s_{2} \leq 2^{-j+1}, 2^{-\ell} \leq s \leq 2^{-\ell+1} .
$$

We also considered the sum $T=\sum_{i, j, \ell=1}^{\infty} T_{i, j, \ell}$. What was proved in [7, Section 9] was that

$$
\left\|T_{i, j, \ell}\right\|_{2 \rightarrow 2}=O\left(e^{-c(i+j+\ell)}\right) .
$$

The proof of (5.5) in [7] served as prototype for the proofs of Section 4.2. But the situation in [7] was simpler.

Then in [7, Section 9] I used Section 5.1 to conclude

$$
\left\|T_{i, j, \ell}\right\|_{p \rightarrow p}=O\left(e^{-c_{p}(i+j+\ell)}\right)
$$

and therefore also that $T$ is $L^{p} \rightarrow L^{p}$ bounded. 
To see this let us denote by $Q(x, y ; h)=Q\left(x, y ; h \mid s_{1}, s_{2}, s\right)$ the integrand in (5.4). Here we identify the operator with its kernel i.e. with a function in $x, y \in \mathbb{R}^{n}$. It is easy to see that if $\Omega^{*}=M \Omega$ is the Hardy-Littlewood maximal function of $\Omega$ on the unit sphere, then for $h=1$ we have

$$
\begin{aligned}
Q(x, y ; 1) & \leq C \Omega^{*}\left(\frac{x-y}{|x-y|}\right) \mathbf{I}(|x-y| \leq C) ;\left|\nabla_{x} Q\right|+\left|\nabla_{y} Q\right|(x, y ; 1) \\
& \leq C s_{1}^{-c} s_{2}^{-c} \mathbf{I}(|x-y| \leq C) .
\end{aligned}
$$

We can scale the above and obtain the corresponding estimates for all $h>0$. These estimates can be integrated in $d h / h$ and we obtain

$$
\begin{aligned}
\left|K_{i, j, \ell}(x, y)\right| & \leq C \Omega^{*}\left(\frac{x-y}{|x-y|}\right)|x-y|^{-n} ; \\
\left|\nabla K_{i, j, \ell}(x, y)\right| & \leq C e^{c(i+j)}|x-y|^{-n-1},
\end{aligned}
$$

for the kernels of the operators $T_{i, j, \ell}$. When $\Omega \in L^{\infty}$ we can combine (5.2) with the above to conclude that the $L_{1} \rightarrow L_{1, \infty}$ norm of $T_{i, j, \ell}, T_{i, j, \ell}^{*}$ are $O\left(e^{\delta(i+j)}\right)$ for an arbitrary $\delta>0$. This interpolated with (5.5) (and dualized) gives (5.6).

For the general case $\Omega \in L^{r}(r>1)$ the previous argument still works because, with the above notation, in (5.3), (5.2) we have

$$
C_{4} \leq C e^{\delta(i+j)},
$$

(for any $\delta>0$ and where $C$ only depends on $n, \delta$ and the constants of (5.7)) .

To see this we can estimate the integrals (5.3) in the range $2^{m}\left|x-x^{\prime}\right| \leq|x-y| \leq$ $2^{m+1}\left|x-x^{\prime}\right|$ in the two different ways by using either the first or the second estimate in (5.7). We then take a geometric average.

\subsection{Proof of Lemma 4.1}

We shall apply the same strategy as in the previous section to the operators $\mathbf{T}_{i, j, \ell}$ of Section 4. Towards this let us denote

$$
\mathbf{Q}\left(x, y ; h \mid s_{1}, s_{2}, s\right)=\left\{\left(I-R_{2^{k}}\right) \check{Q}_{s_{2} h}\{2 \mid s, h\} \check{Q}_{s_{1} h}\left[h>2^{k}\right]\right\}_{k \in \mathbb{Z}} \in \ell^{2},
$$

where each coordinate of $\ell^{2}$ in (5.8) denotes a scalar kernel as above. This after integration in the range (4.5)' gives the kernel of $\mathbf{T}(h \mid i, j, \ell)$. We aim to prove the following estimates

$$
\begin{gathered}
\left|\mathbf{Q}\left(x, y ; 1 \mid s_{1}, s_{2}, s\right)\right| \leq C\left(1+\sigma_{1}+\sigma_{2}+\sigma\right)^{c} \Omega^{*}\left(\frac{x-y}{|x-y|}\right) \mathbf{I}(|x-y| \leq C) ; \\
\left(\left|\nabla_{x} \mathbf{Q}\right|+\left|\nabla_{y} \mathbf{Q}\right|\right)\left(x, y ; 1 \mid s_{1}, s_{2}, s\right) \leq C e^{c\left(\sigma_{1}+\sigma_{2}+\sigma\right)} \mathbf{I}(|x-y| \leq C) \\
x, y \in \mathbb{R}^{n}, s_{j}=e^{-\sigma_{j}} \text { as in Section } 4.3 \sigma, \sigma_{j}>0
\end{gathered}
$$


and where $\Omega^{*}=M \Omega$ is as in (5.7). Once (5.9) has been proved we proceed as in Section 5.2. More explicitly we first scale to obtain the analogous estimates for (5.8) with $h>0$. We then integrate to obtain the analogue of the estimate (5.7) for $\mathbf{K}_{i, j, \ell}$ the kernel of $\mathbf{T}_{i, j, \ell}$ :

$$
\begin{aligned}
\left|\mathbf{K}_{i, j, \ell}(x, y)\right| & \leq C(i+j+\ell)^{c} \Omega^{*}\left(\frac{x-y}{|x-y|}\right)|x-y|^{-n} ; \\
\left|\nabla \mathbf{K}_{i, j, \ell}(x, y)\right| & \leq C e^{c(i+j+\ell)}|x-y|^{-n-1} .
\end{aligned}
$$

We proceed as in Section 5.2 to obtain

$$
\left\|\mathbf{T}_{i, j, \ell}\right\|_{p \rightarrow p}=O\left(e^{-c_{p}(i+j+\ell)}\right) .
$$

Here the presence of the factor $(i+j+\ell)$ in the size estimate (5.10) is easily seen to make no difference. From (5.11) we obtain the boundedness of $\mathbf{T}: L^{p} \longrightarrow L^{p}\left(\ell^{2}\right)$ for the component of $\mathbf{T}$ in the decomposition Section 4.1 coming from (4.5). The other components of $\mathbf{T}$ in the decomposition Section 4.1 are treated identically and they are simpler. This gives the proof of the lemma.

\subsection{Proof of the estimates (5.9)}

For both the size and the gradient estimate in (5.9) what has to be estimated is the square root of:

$$
\sum_{2^{k} \leq 1}\left|\left(I-R_{2^{k}}\right) \check{Q}_{s_{2}}\{2 \mid s, 1\} \check{Q}_{s_{1}}\right|^{2},
$$

and for simplicity, and to illustrate the issue, assume first that $\Omega \in L^{\infty}$. Then, by the compactness of the support of $Q$, in (5.9) we just have to prove the uniform estimate in $x, y$.

Clearly (since $\{\cdots\} \leq Q(x-y)$ ) each individual term in (5.12) is uniformly bounded. Observe however that for the gradient estimate an additional factor $s_{1}^{-1}$ or $s_{2}^{-1}$ will appear. Now to take the summation in $k$ we decompose as we did in Section 4.3 in the two ranges $\left[2^{k} \leq s_{2}\right],\left[s_{2} \leq 2^{k} \leq 1\right]$ and for the first range, as in Section 4.3, we can improve the above uniform estimate by the factor $\frac{2^{k}}{s_{2}}$. The estimate (5.12) as in (5.9) follow and the factor $\left|\log s_{2}\right| \sim\left|\left(k: 2^{k} \geq s_{2}\right)\right|$ comes from the second range.

For the general case $\Omega^{*} \in L^{1}$ the proof is essentially the same:

To estimate the uniform norm of $\check{Q}_{s_{i}} * Q$ we have to use $\|\Omega\|_{1}$ multiplied with the $L^{\infty}$-norms of $\check{Q}_{s_{1}}, \check{Q}_{s_{2}}$ (or at least of one of them!). This accounts for the $s_{1}^{-c} s_{2}^{-c}$ in the constant of the gradient estimate of (5.9).

To obtain the size estimate in (5.9) we do not use $\left\|\check{Q}_{s_{1}}\right\|_{\infty}$ but dominate each term of (4.12) by $\Omega^{*}\left(\frac{x-y}{|x-y|}\right)$ with the same convergence factor $\frac{2^{k}}{s_{2}}$ in the range $2^{k} \leq s_{2}$. This completes the proof of (5.9). 


\section{Appendix}

\section{A.1. (Carleson measures)}

We recall the notation of $[7,(3.15)]$ so that in (3.6) we have

$$
\begin{gathered}
G_{h}(x, y)=\left|[a]_{x, y}-\check{P}_{h} a(x)\right| ; \\
\beta_{\sigma}^{2}(x, h)=\int\left|Q_{h}(x-y)\right| G_{h}^{2}(x, y) \sigma(y) d y ; \\
a \in C_{0}^{\infty}, \sigma \geq 0 .
\end{gathered}
$$

I pointed out in the remark of [7, Section 3.6] that with $G^{2}(y)=\int G_{h}^{2}(x, y) \frac{d x d h}{h}$ we have

$$
\|G\|_{2 r} \leq C\|a\|_{2 r} ; r>1
$$

This was seen as follows. For $g \in C_{0}^{\infty}$ we have

$$
\int G^{2}(y) g(y) d y \leq \iiint \beta_{\sigma}^{2}(x, h) \frac{d x d h}{h} \leq C \int|a(x)|^{2} \sigma^{* *}(x) d x,
$$

where $\sigma=\left(M g^{1+\epsilon}\right)^{\frac{1}{1+\epsilon}}$ for some $\epsilon>0$ with $\frac{q}{1+\epsilon}>1$ and $\frac{1}{q}+\frac{1}{r}=1$ as in [7, lemma, Section 3.5]. As for $\sigma^{* *}$ it was constructed in that lemma so that $\left\|\sigma^{* *}\right\|_{q} \leq$ $C\|g\|_{q}$ and such that (A.2) holds. (A.1) is a consequence of (A.2).

From this we obtain the following Carleson measure property:

$$
\iint_{T(I)} \beta_{\sigma}^{2}(x, h) \frac{d x d h}{h} \leq C \sigma(I)\|a\|_{\infty}^{2} ; \text { for a cube } I \subset \mathbb{R}^{n},
$$

where $T(I)$ is the tent above I $(c f .[6,7])$ and $\sigma \in A_{\infty}$ is arbitrary. To see this, by the compactness of the support of $Q$ in the left hand side of (A.3), we can localize $a$ and replace it by $a_{1}=\chi_{I_{1}} a$ for some larger concentric $I_{1} \supset I$. It follows that the left hand side of (A.3) can be dominated by $\int G_{1}^{2}(y) \sigma(y) d y$ where $G_{1}$ is constructed exactly as $G$ but where we use the localized $a_{1}$. We then use (A.1) on $G_{1}, a_{1}$ and the reverse Hölder inequality (3.1) on $\sigma$, and we obtain (A.3). The Property 3.3 of Section 3 is a consequence of (A.3) and the general theory $c f$. [6].

[This is a good place to point out that there is an error in the statement, but not in the proof, of [7, lemma, Section 3.5]: $\sigma^{* *}=\left(\sigma_{\alpha}\right)^{1 / \alpha}$ and not $\left(\Omega^{*} \sigma^{\alpha}\right)^{1 / \alpha}$. This error is purely notational and makes no difference either in [7] or here.] 


\section{A.2. (Kernels)}

\section{The scalar kernel}

For the convenience of the reader I will start with rerunning the argument of [7, Section 2.6] for the kernel (0.7):

$$
Q(x, y ; h)=\Omega\left(\frac{x-y}{|x-y|}\right) \theta_{h}(|x-y|) F\left([a]_{x, y}\right) .
$$

We consider

$$
\widetilde{Q}(x, y ; h)=\check{P}_{h} Q(x, y ; h)=\int \check{P}_{h}(x-z) Q(z, y ; h) d z,
$$

and define analogously $Q_{s}(x, y ; h)=\check{Q}_{s h} Q$ for $0<s<1$. What was needed and proved in [7] is the following:

Lemma. Let the notation be as above. Then:

(i) If we assume that $\widetilde{Q}: D_{2} \rightarrow L_{2}$ bounded then $\widetilde{Q}: D_{\infty} \rightarrow B M O$ is bounded.

(ii) If we assume that $Q_{s}: D_{2} \longrightarrow L_{2}$ bounded uniformly in $s$ the $D_{\infty} \longrightarrow$ $B M O$ operator norm of $Q_{s}$ is $O\left(s^{-\lambda}\right)$ for any $\lambda>0$.

(iii) If we assume that the $D_{2} \longrightarrow L_{2}$ norm of $Q_{s}$ is $O\left(s^{\epsilon}\right)$ for some $\epsilon>0$ then the $D_{p} \longrightarrow L_{p}$ norm of $Q_{s}$ is $O\left(s^{\epsilon_{p}}\right)$ for some $\epsilon_{p}>0(1<p<\infty)$.

Part (iii) for $2 \leq p<\infty$ is an immediate consequence of (ii) and interpolation, (cf. Appendix 3 below). For the case $1 \leq p \leq 2$ we interpolate between $p=1$ and 2 and we do not need (ii). In the above lemma and throughout I abusively use the same notation for the kernel and the induced mapping (0.6).

The proof of (i) and of (ii) in the special case $\Omega \in L^{\infty}$ are easy: Indeed we have

$$
\begin{aligned}
& |\widetilde{Q}(x, y ; h)|,\left|Q_{s}(x, y ; h)\right| \leq C h^{-n} \mathbf{I}(|x-y| \leq c h), \\
& \left|\nabla_{x} \widetilde{Q}(x, y ; h)\right| \leq C h^{-n-1} \mathbf{I}(|x-y| \leq c h), \\
& \left|\nabla_{x} Q_{s h}(x, y ; h)\right| \leq C s^{-1} h^{-n-1} \mathbf{I}(|x-y| \leq c h) .
\end{aligned}
$$

In particular for all $0<\lambda<1$ it follows that:

$$
\left|Q_{s h}(x, y ; h)-Q_{s h}\left(x^{\prime}, y ; h\right)\right| \leq C s^{-\lambda} h^{-\lambda-n} \mathbf{I}(|y| \leq C h) ;|x|,\left|x^{\prime}\right| \leq 1,|y|+h \geq C,
$$

and as a consequence:

$$
\int_{|y|+h \geq C}\left|Q_{s h}(x, y ; h)-Q_{s h}\left(x^{\prime}, y ; h\right)\right| \frac{d y d h}{h} \leq C s^{-\lambda} ; \lambda>0,|x|,\left|x^{\prime}\right| \leq 1 .
$$


The analogous estimate for $\widetilde{Q}$ holds for $\lambda=0$. If we now use the hypothesis of the lemma and the previous estimate, together with the cut off properties of [7, Section $2.1]$, we deduce the required BMO property for $\widetilde{Q} A, Q_{s} A\left(A \in D_{\infty}\right)$ tested on the unit cube. The situation is clearly dilation and translation invariant and our result follows.

When $\Omega \in L^{r}$ with $r<+\infty$ nothing changes in the estimates for $\widetilde{Q}$ and for $Q_{s}$ we have

$$
\begin{gathered}
\left|Q_{s}(x, y ; h)\right| \leq C h^{-n} \Omega^{*}\left(\frac{x-y}{|x-y|}\right) \mathbf{I}(|x-y| \leq c h), \\
\left|\nabla_{x} Q_{s}(x, y ; h)\right| \leq C h^{-n-1} s^{-a} \mathbf{I}(|x-y| \leq c h) ; a=a(n, r)>0,
\end{gathered}
$$

where $\Omega^{*}=M_{\Sigma} \Omega$ is the Hardy-Littlewood maximal function of $\Omega$ on $\Sigma$. We shall then proceed as before and denote by $f(x)=\int_{|y|+h>C} Q_{s}(x, y, h) A(y, h) \frac{d y d h}{h}$ for some $A \in L^{\infty}$ with norm 1 . For the BMO condition tested on the unit cube $I$ it suffices therefore to estimate

$$
\begin{gathered}
\iint_{\left|x_{i}\right| \leq 1}\left|f\left(x_{1}\right)-f\left(x_{2}\right)\right| d x_{1} d x_{2} \leq \iiint_{\substack{(y, h) \in T \\
\left|x_{i}\right| \leq 1}}\left|Q_{s}\left(x_{1}, y ; h\right)-Q_{s}\left(x_{2}, y ; h\right)\right| \frac{d x_{1} d x_{2} d y d h}{h} \\
T=\bigcup_{j \geq 100} T_{j} ; T_{j}=\left[2^{j}<h<2^{j+1} ;|y| \leq C 2^{j}\right] ; j \geq 1 .
\end{gathered}
$$

The integration range $T$ holds because of the compactness of the support of $Q$. Using the two estimates (A.6) separately on each $T_{j}$ and taking a geometric mean and summing we see that we can estimate (A7) by $C s^{-\lambda}\|A\|_{\infty} 0<s<1$ for any $\lambda>0$. This completes the proof of the lemma.

\section{The vector valued kernel}

We shall examine now the vector valued kernel:

$$
\widetilde{\mathbf{Q}}(x, y ; h)=\left\{\left(I-R_{2^{k}}\right) \widetilde{Q}(x, y ; h) \mathbf{I}\left(2^{k}<h\right)\right\}_{k} \in H=\ell^{2},
$$

and the analogue $\mathbf{Q}_{s}$ where in $\{\cdots\}$ we replace $\widetilde{Q}$ by $Q_{s}$. The analogue of the estimates (A.5) for $\widetilde{\mathbf{Q}}$ also hold and this is essentially trivial to verify by the smoothness of $\check{P}_{h}$ and the geometric decay of the diameters of the supports of $I-R_{2^{k}}$. The analogue of (A.6) for $\mathbf{Q}_{s}$ is

$$
\begin{aligned}
& \left|\mathbf{Q}_{s}(x, y ; h)\right|_{H} \leq C h^{-n}(1+|\log s|) \Omega^{*}\left(\frac{x-y}{|x-y|}\right) \mathbf{I}(|x-y| \leq c h) ; \\
& \left|\nabla_{x} \mathbf{Q}_{s}(x, y ; h)\right|_{H} \leq C h^{-n-1} s^{-a} \mathbf{I}(|x-y| \leq c h) .
\end{aligned}
$$

The difference with $\widetilde{\mathbf{Q}}$ lies simply in the fact that in the $\ell^{2}$-coordinates $k$ of (A.8) we have to distinguish two ranges: range- $1=\left[2^{k}<s h\right]$ and range $2=\left[s h<2^{k}<h\right]$. 
For the first range the same argument as for $\widetilde{\mathbf{Q}}$ works and nothing changes. For range 2 we simply use the fact that the cardinality of range 2 is $\sim 1+|\log s|$ and estimate separately each individual coordinate. We shall omit the details which are similar to what was done in Section 4.3 and Section 5.4.

It is clear now that in the proof of the lemma we have room to absorb the logarithms. The conclusion is therefore that the lemma holds verbatim for the vector valued kernels $\widetilde{\mathbf{Q}}$ and $\mathbf{Q}_{s}$.

\section{A.3. (Interpolation)}

The interpolation that we used in the proof of Appendix 2 Lemma (iii) says this: Let $T$ be a linear operator and assume that:

$$
\begin{aligned}
& T: D_{\infty} \longrightarrow B M O\left(\ell^{2}\right) \text { with norm }\|T\|_{(\infty)} ; \\
& T: D_{2} \longrightarrow L^{2}\left(\ell^{2}\right) \text { with norm }\|T\|_{(2)},
\end{aligned}
$$

then

$$
\begin{aligned}
& T: D_{p} \longrightarrow L^{p}\left(\ell^{2}\right) ; \frac{1}{p}=\frac{\theta}{2}, 0<\theta<1, \\
& \text { with norm }\|T\|_{(p) \leq C_{\theta}\|T\|_{(2)}^{\theta}\|T\|_{(\infty)}^{1-\theta}}^{1-} .
\end{aligned}
$$

One way to see this is to use $K$-real interpolation and use the facts

$$
D_{p} \subset\left[D_{\infty}, D_{2}\right]_{\theta, p} ;\left[B M O\left(\ell^{2}\right), L^{2}\left(\ell^{2}\right)\right]_{\theta, p} \subset L^{p}\left(\ell^{2}\right) \text {. }
$$

The proof of the first fact in (A.11) has been spelled out in [7, Appendix]. This proof follows standard lines anyway.

For the second fact in (A.11) one can use duality and reduce it to vector valued Hardy spaces ( $c f$. O. Blasco \& Q. Xu: J. of Func. Analysis 102 (1991) pages 191194) and prove the fact:

$$
L^{p}\left(\ell^{2}\right) \subset\left[L^{2}\left(\ell^{2}\right), H^{1}\left(\ell^{2}\right)\right]_{p, \theta} .
$$

For the proof of (A.12) we can use the same proof as for the scalar case:

P. Jones: The "Zygmund Conference" Wadworth 1981.

J. Peetre: Studia Math. T. LXI, 1979 pages 191-194.

The duality and the use of the $H^{1}$-spaces has been avoided by J.-L. Journé (L.N.M. No. 994, Springer, page 40). Journé uses directly the sharp function in a very analogous context. With the same method we can deduce directly from (A.9) that $D_{p} \longrightarrow L_{p, \infty}\left(\ell^{2}\right)$ is bounded. From this a slightly weaker version of (A.10) follows. This is sufficient for Lemma (iii) in Appendix 2. 


\section{References}

[1] M. Christ and J.-L. JOURnÉ, Polynomial growth estimates for multilinear singular operators, Acta Math. 159 (1987), 51-80.

[2] R. R. Coifman, Y. Meyer and E. M. Stein, Some new function spaces and their applications in harmonic analysis, J. Funct. Anal. 62 (1985), 302-335.

[3] B. E. DAhlBerg, Poisson semigroups and singular integrals, Proc. Amer. Math. Soc. 97 (1986), 41-48.

[4] J. DuAndikoetxea and J. L. Rubio De Francia, Maximal and singular integral operators via Fourier transform estimates, Invent. Math. 84 (1986), 541-561.

[5] J. Garcia-Cuerva, An extrapolation theorem in the theory of $A_{p}$ weights, Proc. Amer. Math. Soc. 87 (1983), 422-426.

[6] E. M. Stein, "Harmonic Analysis Real-Variable Methods, Orthogonality, and Oscillatory Integrals", Princeton University Press, 1993.

[7] N. Th. VAropoulos, Singular integrals and potential theory. Milan J. Math. 75 (2007), $1-60$.

[8] N. Th. Varopoulos, Singular integrals and potential theory (II), Milan J. Math. 76 (2008), 419-429.

I.U.F. Université Paris VI

and

Dipartimento di Matematica e Applicazioni Università di Milano Bicocca

Via Cozzi, 53

20125 Milano, Italia 